\title{
Zingiber officinale extract and omega-3 fatty acids ameliorate endoplasmic reticulum stress in a nonalcoholic fatty liver rat model
}

\author{
Mohamed A. Kandeil $^{1}$ | Reem M. Hashem ${ }^{2}$ | Mohamed O. Mahmoud ${ }^{2}$ | \\ Mona H. Hetta ${ }^{3}$ | Mohamed A. Tohamy 2,4
}

${ }^{1}$ Department of Biochemistry, Faculty of Veterinary Medicine, Beni-Suef University, Beni-Suef, 62511, Egypt

${ }^{2}$ Department of Biochemistry, Faculty of Pharmacy, Beni-Suef University, Beni-Suef, 62514, Egypt

${ }^{3}$ Department of Pharmacognosy, Faculty of Pharmacy, Fayoum University, Fayoum, 63514, Egypt

${ }^{4}$ Department of Molecular and Integrative Physiology, University of Michigan, Ann

Arbor, 48109, MI, USA

\section{Correspondence}

Mohamed A. Tohamy, Department of Biochemistry, Faculty of Pharmacy, BeniSuef University, Beni-Suef 62514, Egypt.

Email:mtohamy@med.umich.edu,

Mohamed011157@pharm.bsu.edu.eg

Funding information

This work was supported by the Support and Project Finance Office, Scientific Research Developing Unit, Beni-Suef University, Beni-Suef, Egypt [grant numbers YRP32, 2016/2017, Grant holder (PI): Mohamed A. Tohamy]

\begin{abstract}
Endoplasmic reticulum (ER) stress was reported to play a major role in non-alcoholic fatty liver disease (NAFLD) induction and progression. Here, we study the effect of Zingiber officinale and omega-3 fatty acids on ER stress for treating NAFLD. Male Wistar rats were fed on a normal diet (control group) or high-fat diet (HFD) for 8 weeks. The HFD rats were later treated with vehicle, omega-3 or with Z. officinale extract. HFD group demonstrated significantly more body weight gain and higher plasma lipid profile, glucose, and hepatic enzymes. The expressions of lipogenic ChREBP and ER stress genes CHOP, XBP1, and GRP78 were increased. This was accompanied by intrahepatic fat accumulation visualized by hepatic morphology and H\&E-stained sections. Treatment with Z. officinale and omega- 3 fatty acids reverted these changes into a normal healthy state. From these results, we prove that both therapeutic approaches can be potential drugs for treating NAFLD besides other ER stress-associated diseases.

\section{Practical applications}

The effect of Zingiber officinale extract and omega-3 fatty acid on ER stress associated with NAFLD was investigated. The results revealed that $Z$. officinale extract and omega- 3 fatty acids significantly inhibited ER stress and intrahepatic fat accumulation with the upper hand for Z. officinale extract. Both can be used as future promising therapies for the treatment of NAFLD patients and also treating different diseases that involve ER stress as a pathological modulator like diabetes mellitus, Alzheimer's disease, Parkinson's disease, and cancer.
\end{abstract}

\section{KEYWORDS}

ER stress, high-fat diet, NAFLD, omega-3, steatosis, Zingiber officinale

\section{1 | INTRODUCTION}

Non-alcoholic fatty liver disease (NAFLD) is one of the most prevalent diseases worldwide. It became a research interest for many investigation groups. NAFLD is considered the most abundant reason for abnormal hepatic function in the United States as it affects around 30 million Americans. NAFLD prevalence in the general population is estimated between $14 \%-24 \%$ and $20 \%-30 \%$ in adults with a higher incidence in industrial countries (Browning \& Horton, 2004; Buzzetti, Pinzani, \& Tsochatzis, 2016). 
Its effect can be as simple as simple steatosis but can develop into more serious nonalcoholic steatohepatitis, hepatic fibrosis, liver cirrhosis, hepatocellular carcinoma, and increased mortality (PisoneroVaquero et al., 2015). Obesity, insulin resistance (IR), metabolic syndrome, and type 2 diabetes are considered as risk factors that increase the prevalence of the NAFLD pathogenesis (Lai et al., 2016).

The endoplasmic reticulum (ER) is a major site for correct protein folding. However, during cellular stress conditions like high-fat diet (HFD) feeding, the folding capacity of ER is surpassed and ER stress markers are elevated (Zhang, Xue, Zhang, Yang, \& Shi, 2012). To restore ER to a normal state, an intracellular pathway named unfolded protein response is stimulated. This pathway is triggered to initially attenuate protein synthesis but if ER stress is not resolved, cellular death is induced, and the pathogenesis of many diseases including NAFLD is stimulated and progressed to more serious states (Tirosh, 2014). Thus, ER stress is proposed to play a crucial role in the pathologies of NAFLD.

Many therapeutic trends were investigated to modulate NAFLD pathogenesis. The use of natural products and nutritional therapy for many health problems has become the interest of many researchers. Some reviews showed several examples of the use of phytochemicals like polyphenols and flavonoids and their ability to modulate the molecular pathway involved in NAFLD progression (Dongiovanni, Lanti, Riso, \& Valenti, 2016; Mazidi, Katsiki, \& Banach, 2019; Rodriguez-Ramiro, Vauzour, \& Minihane, 2016). The rhizomes of Zingiber officinale have shown many therapeutic activities ranging from antitumor, neuroprotective, anti-inflammatory, antibacterial, gastroprotective, and antidiabetic through its effect on genetic and metabolic activities(Rahmani, Al Shabrmi, \& Aly, 2014; Wang, $\mathrm{Ke}, \mathrm{Bao}, \mathrm{Hu}, \&$ Chen, 2017). Omega-3 fatty acids are found to have a good therapeutic effect against Obesity, IR, and type 2 diabetes mellites. Other studies showed its effect in lowering lipogenic genes, oxidative stress, and inflammation (Denny Joseph \& Muralidhara, 2012; Molinar-Toribio et al., 2015).
In this study, we tried to explore the effect of polyphenol-rich $Z$. officinale extract against ER stress for the treatment of experimental NAFLD model induced in Wistar albino rats in comparison to commercial omega-3 fatty acids.

\section{MATERIALS AND METHODS}

\subsection{Preparation of Z. officinale ethanolic extract}

Authenticated fresh rhizomes of $Z$. officinale Roscoe, Zingiberaceae, have been obtained from El-Orman garden, Egypt. The authentication has been performed by the Department of Pharmacognosy, Faculty of Pharmacy, Fayoum University under the supervision of Professor Mona $\mathrm{H}$. Hetta with a voucher number (FP-01). Two Kilograms of Z. officinale rhizomes were crushed and shaken with $3 \mathrm{~L}$ of ethanol (95\%) for $48 \mathrm{hr}$ at room temperature. The ethanolic extract distilled off using a rotary evaporator "Stuart, Staffordshire, UK" at $55^{\circ} \mathrm{C}$ (Singh, Akanksha, Singh, Maurya, \& Srivastava, 2009), under reduced pressure affording a brown semisolid residue (Figure 1).

\section{2 | Z. officinale phytochemical analysis}

Phytochemical screening for carbohydrates (Molisch's and Fehling's tests), terpenoids (Salkowski test), saponins (Froth test), tannins ( $\mathrm{FeCl}_{3}$ and Lead acetate tests), flavonoids (Sodium hydroxide and Sulfuric acid tests), and alkaloids (Dragendorff's reagent test) have been carried out according to the previous publications (Bhargava, Dhabhai, Batra, Sharma, \& Malhotra, 2012; N, 2011).

Total phenolic contents from $Z$. officinale extract were determined using the Folin-Ciocalteu method (Pan, Su, Cai, \& Wu, 2017; Stanković, 2011). Briefly, standard concentrations of gallic acid (25, $50,75,100,125$, and $150 \mu \mathrm{g} / \mathrm{ml}$ ) besides the extract samples were prepared in methanol solvent in triplicates. Then, the samples were

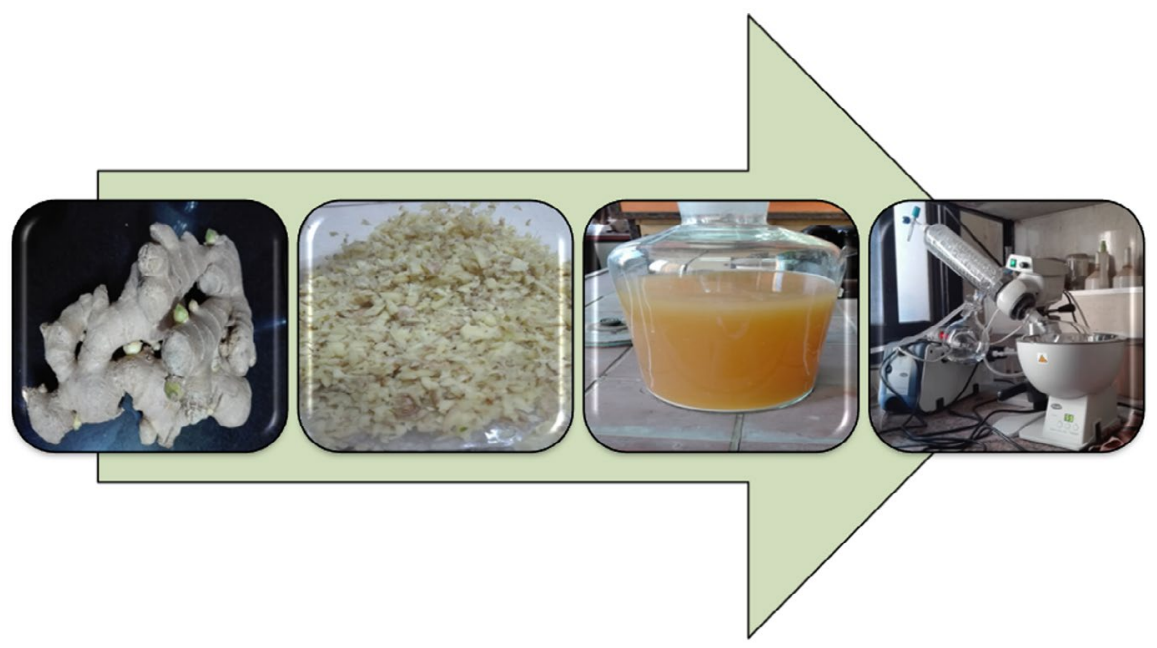

FIGURE 1 Preparation of ginger extract 


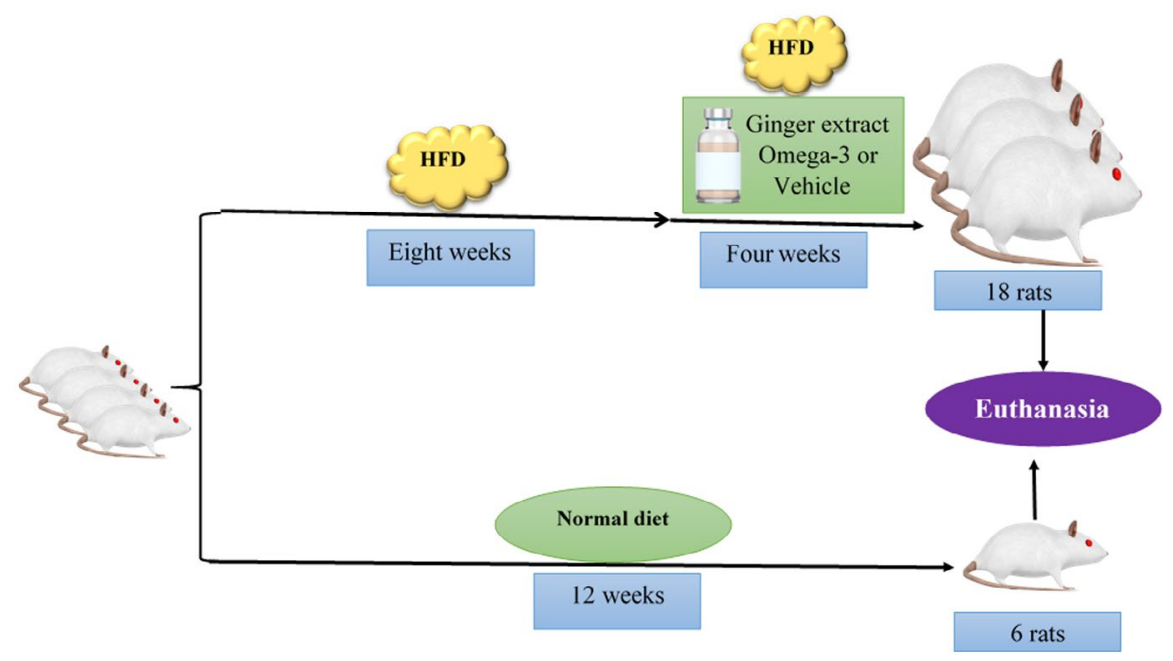

FIGURE 2 Experimental design

incubated with Folin-Ciocalteu reagent at $40^{\circ} \mathrm{C}$ for $60 \mathrm{~min}$. The absorbances of the samples were measured at $765 \mathrm{~nm}$ against the blank using a spectrophotometer "Shimadzu, Japan." Regression curve was plotted and the total phenolic content in Z. officinale extract residue has been calculated and expressed in terms of $\mu \mathrm{g}$ of gallic acid equivalent (GAE) per mg of Z. officinale extract.

\section{3 | Animals}

Twenty-four male Wistar rats (Rattus norvegicus) with body weight around $100 \mathrm{~g}$ were obtained from the animal care unit in the Faculty of Pharmacy - Beni-Suef University, Egypt.

Animals were housed in plastic cages under a controlled environmental condition in an air-conditioned room with temperature of $22 \pm 5^{\circ} \mathrm{C}$ for 12 -hr light-dark cycles. The animals had free access to food and water. The experimental procedure started directly after 2 weeks for acclimation and lasted for 12 weeks. All animal experiments, conditions, and procedures comply with international ethical guidelines relied on the Guide for the Care and Use of Laboratory Animals, 8th Edition (Council, 2011) and adopted previously by AAALAC International. The laboratory work protocols have been revised and approved by Beni-Suef University International Animal Care and Use Committee (BSU_ IACUC) with permit number 018-59.

In the first phase (NAFLD induction phase) of the experiment, 18 rats were fed on HFD and high sugar diet for 8 weeks. The remaining six rats were fed on a normal calories diet to serve as normal healthy controls. After the NAFLD induction, the rats fed on HFD were divided into three equal groups randomly (Figure 2). All three subgroups fed with HFD were treated with either water as a vehicle, commercial omega-3 fatty acids (800 mg/Kg BW-Sedico, Egypt) (Marsman et al., 2011), or Z. officinale extract dissolved in water vehicle $(200 \mathrm{mg} / \mathrm{kg} \mathrm{BW})$ (Bhandari, Kanojia, \& Pillai, 2005; Nammi, Sreemantula, \& Roufogalis, 2009) by oral gavage daily for 4 weeks. Omega-3 fatty acids used in the experiment was an oily liquid form composed of eicosatetraenoic
TABLE 1 Diet for normal control and HFD rats

\begin{tabular}{|lll|}
$\begin{array}{l}\text { Diet } \\
\text { composition }\end{array}$ & $\begin{array}{l}\text { Normal control rats' } \\
\text { diet }\end{array}$ & HFD rats' diet \\
\hline Food (w/w) & $\begin{array}{l}55-70 \% \\
\text { carbohydrates }\end{array}$ & $\begin{array}{c}40 \% \text { Normal Control rats' } \\
\text { diet (chow) }\end{array}$ \\
& $23 \%$ protein & $30 \%$ beef tallow fat \\
& $6 \%$ fat & $20 \%$ sucrose \\
& $3 \%$ fibers & $9.8 \%$ corn starch \\
& $\begin{array}{c}\text { 1-4\% vitamins/ } \\
\text { minerals }\end{array}$ & $0.2 \%$ bile salts \\
& Plain drinking water & Water contains 10\% \\
& & sucrose and 10\% fructose \\
\hline Drinking & &
\end{tabular}

acid and docosahexaenoic acid in 3:2 percentage and given in its original form. The diet of both groups is described in Table 1.

\subsection{Analysis of plasma parameters and liver histology}

After the animals being fasted for $12 \mathrm{hr}$, the rats were weighed and then blood samples were collected from a retro-orbital vein using heparinized capillary tubes. The blood samples were centrifuged at $1,500 \mathrm{rpm}$ for $30 \mathrm{~min}$. Plasma was withdrawn and divided into three aliquots in Eppendorf tubes to minimize possible thaw/refreeze cycles. One aliquot was used for immediate estimation of fasting plasma glucose level, while other aliquots were stored at $-20^{\circ} \mathrm{C}$ for future analysis. Stored aliquots were used later for analysis of triglycerides (TG), total cholesterol (TC) levels besides aspartate aminotransferase (AST), and alanine aminotransferase (ALT) activities using "Randox, UK" biochemical kit. Plasma free fatty acids were analyzed according to Itaya, 1977. All readings were measured using "JENWAY, UK" spectrophotometer.

After rat euthanasia by cervical dislocation, the liver was photographed, weighed, then excised, washed with saline, and stored 
in $10 \%$ formalin solution to be used later for hematoxylin and eosin staining. Photomicrographs were taken with Leica full HD camera mounted on a trinuclear Leica microscope.

\section{5 | Detection of ChREBP, CHOP, XBP1, and GRP78 hepatic genes expressions by quantitative polymerase chain reaction}

Total RNA was isolated using Qiagen tissue extraction kit "Qiagen, USA." The extracted RNA ( $1 \mu \mathrm{g})$ was reverse transcribed using cDNA reverse transcription kit "Fermentas, USA." qPCR experiments were performed using SensiFAST ${ }^{\mathrm{TM}} \mathrm{SYBR}^{\circledR} \mathrm{Hi}$-ROX Kit "Bioline Reagents Ltd; UK" and analyzed by Applied Biosystem thermocycler version 3.1 "StepOne ${ }^{\mathrm{TM}}$, USA" using specific primers from "Integrated DNA Technologies, USA."

\section{6 | Statistical analysis}

Statistical analysis and graphs were performed using GraphPad Prism 6.01 "GraphPad Software, Inc., USA" and R version 3.1.2 "The R Project for Statistical Computing." Data were represented as groups means \pm standard errors using one-way ANOVA and Tukey post hoc multiple comparison test.

\section{3 | RESULTS}

\section{1 | Phytochemical screening}

Z. officinale extract revealed the presence of carbohydrates, terpenoids, saponins, tannins, flavonoids, and alkaloids (Figure 3a). Total phenolic content in Z. officinale extract residue was $113.76 \mu \mathrm{g}$ GAE/ $\mathrm{mg} Z$. officinale extract accounting for more than $11 \%$ of the extract weight (Figure 3b).

\section{2 | Biochemical analysis}

By assessing the body weight gain for HFD and normal diet-fed rats, we demonstrated that feeding rats with HFD caused $57 \%$ more body weight gain compared to the control rats kept on a normal diet. After starting omega-3 or Z. officinale extract treatments, rats fed with HFD and treated with either therapy showed a slower increase in body weight. Rats treated with omega-3 showed around 57\% less body weight gain compared to untreated rats fed with HFD. Moreover, rats treated with Z. officinale extract demonstrated $89 \%$ less body weight gain compared to untreated rats fed with HFD (Figure 4).

The HFD-fed rats showed a clear significant increase in blood glucose and lipids profile. There was also a significant elevation in

(a)

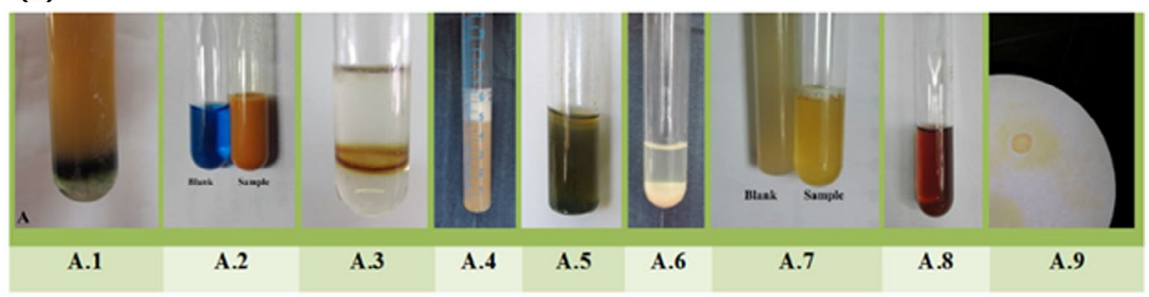

(b) Gallic acid standard curve

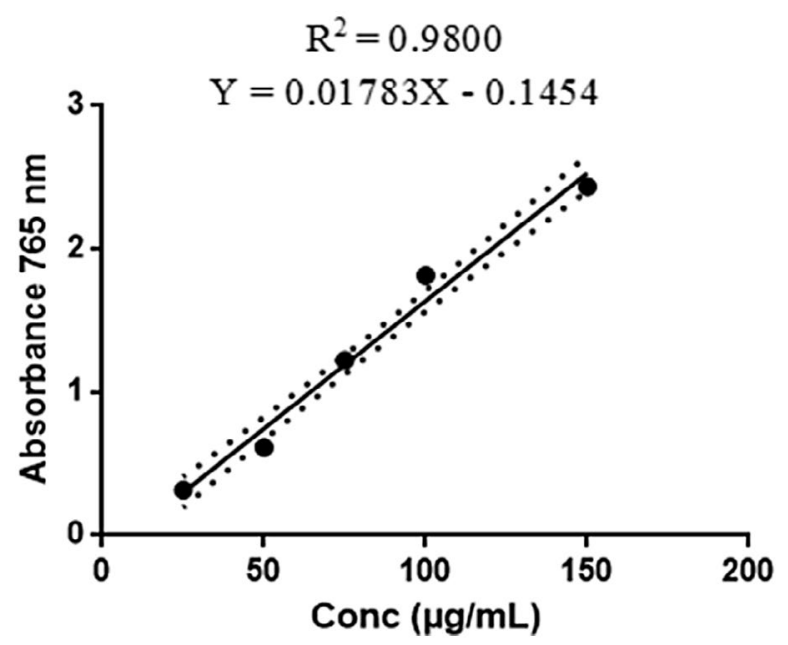

FIG URE 3 (a) Phytochemical screening tests. (A.1) Positive Molisch's, (A.2) Fehling, (A.3) Salkowski, (A.4) Froth, (A.5) FeCl 3 , (A.6) lead acetate, (A.7) $\mathrm{NaOH},(A .8)$ Conc. Sulfuric, (A.9), Dragendorff's reagent tests. (b) Gallic acid standard regression curve 

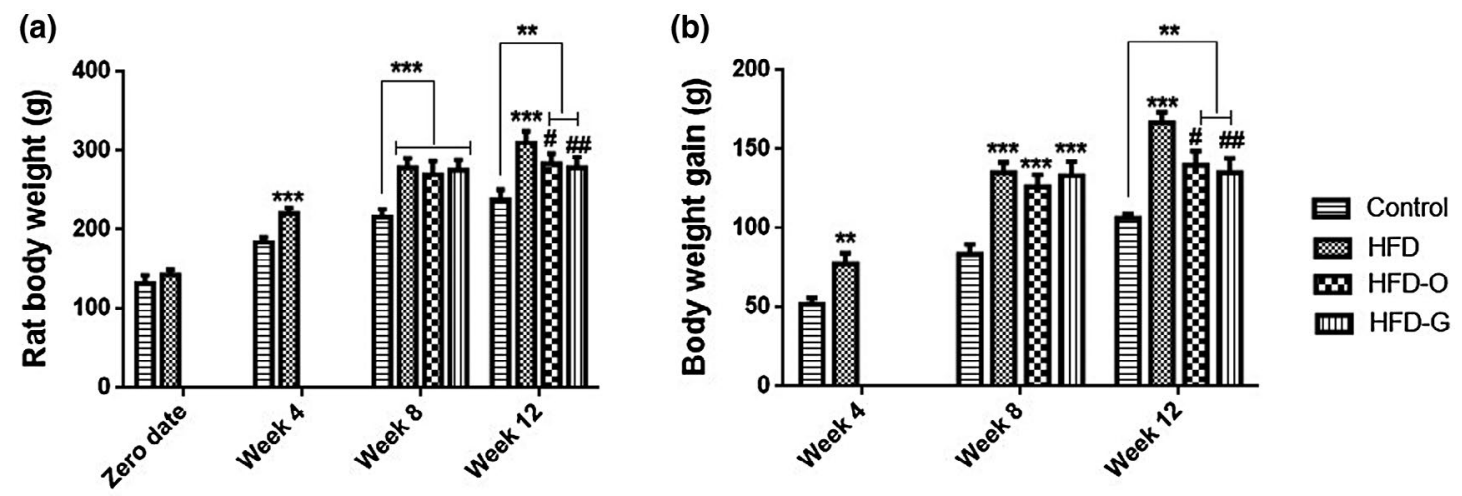

(c)

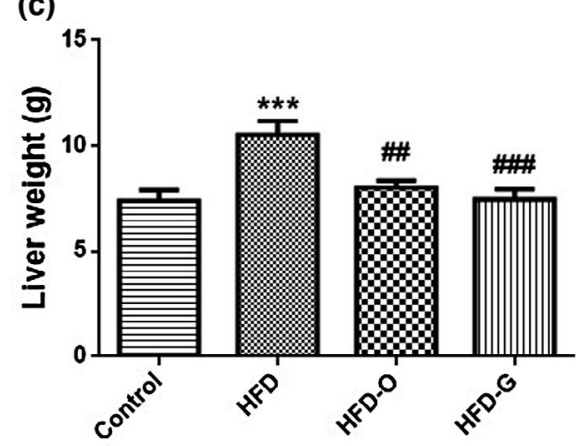

FIG URE 4 (a) Rat body weight (g). HFD group was split equally from the eighth week before treatment until the end of the study. (b) Body weight gain (g). HFD group was split equally from the eighth week before treatment until the end of the study. (c) Liver weight (g). Data are represented as means \pm SE. HFD means are statistically significant at $\left({ }^{*}\right) p \leq .1,\left({ }^{* *}\right) p \leq .05,\left({ }^{* * *}\right) p \leq .001$ compared to control group. HFD-O and HFD-G means are statistically significant at (\#) $p \leq .1$, (\#\#) $p \leq .05$, (\#\#\#) $p \leq .001$ compared to HFD group. HFD, high-fat diet group; HFD-O, high-fat diet group treated with omega-3 fatty acids; HFD-G, high-fat diet group treated with Zingiber officinale extract

plasma hepatic enzymes ALT and AST. These results were coherent with higher liver weight in HFD-fed rats (Figure 4). The treated rats had improved blood biochemical profile through rendering plasma glucose, TG, and TC back to a normal level with non-significant advantage for $Z$. officinale extract over omega-3. The data are represented in (Figure 5) as means \pm standard error (SE).

\section{3 | Relative genes expression levels}

Rats kept on HFD had significant induction of ChREBP, CHOP, XBP1, and GRP78 hepatic genes expressions over the normal rats. Our therapeutic approaches caused a significant correction of the deregulated genes expressions till a non-significant level to the normal group with non-significant advantage for Z. officinale over omega-3 (Figure 6).

\section{4 | Liver morphology and histology}

HFD-fed rats livers had a pale brownish color as a result of fat accumulation. Both the $Z$. officinale-treated and omega-3-treated groups had the normal healthy bright red color of the liver. The liver H\&E-stained sections for the normal rats showed normal hepatic cells status around the central vein. The rats fed with HFD had many micro-vesicular fatty accumulations distributed in almost all the section besides congested sinusoids. The fat droplets in many cells pushed the nucleus to periphery toward forming the signet ring appearance. The treated rats showed less steatosis in both the dispersion and the number of fat globules showing few micro-vesicular fat droplets (Figure 7).

\section{4 | DISCUSSION}

As the global epidemic of obesity fuels metabolic conditions, the clinical and economic burden of NAFLD will become enormous. NAFLD is highly linked with obesity, IR, and metabolic syndrome (Younossi et al., 2016). The pathogenesis of the disease could evolve to more serious hepatic cancer, liver fibrosis, and death risk (Buzzetti et al., 2016; Saponaro, Gaggini, \& Gastaldelli, 2015). There were many studies investigating the effect of drugs on NAFLD besides the possible molecular mechanisms involved in disease pathogenesis/treatment to discover new therapeutic targets (Adams \& Angulo, 2006; Rodriguez-Ramiro et al., 2016). Here we investigate the effect of $Z$. officinale ethanolic extract and omega-3 fatty for the treatment of NAFLD in male Wistar rats via inhibiting of ER stress risk factorassociated parameters.

The administration of HFD resulted in more body weight gain besides significant elevation in plasma lipid profile, glucose, and 
(a)

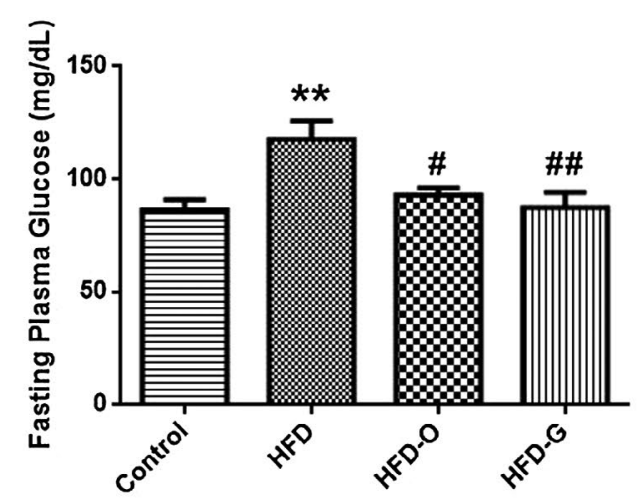

(c)

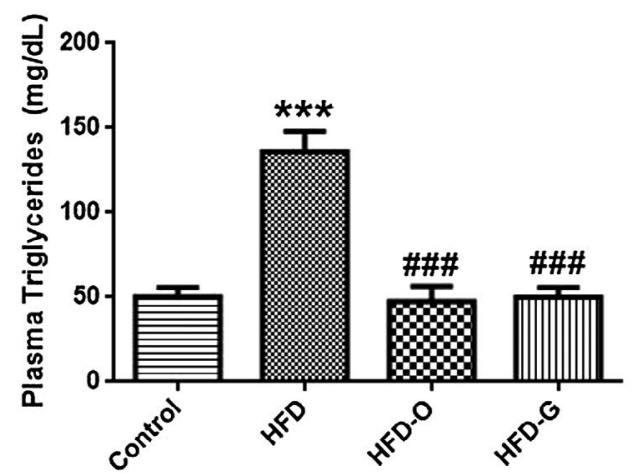

(e)

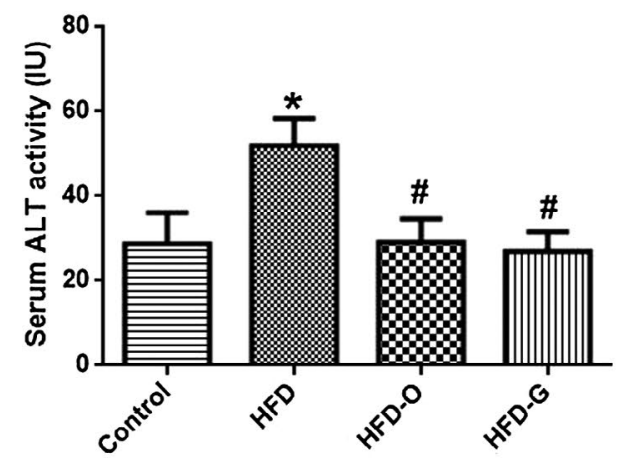

(b)

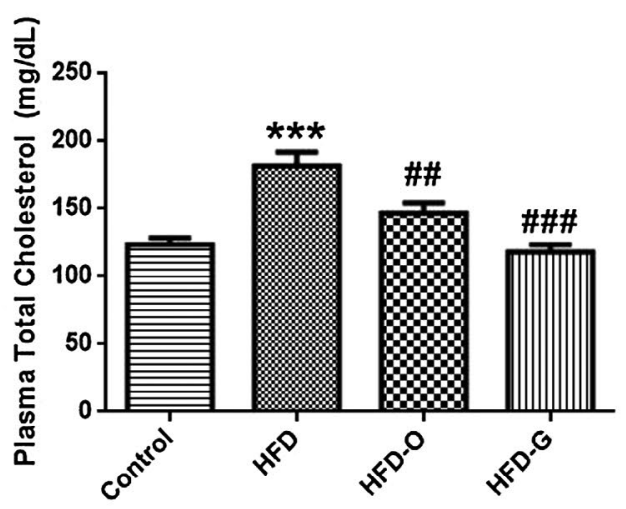

(d)

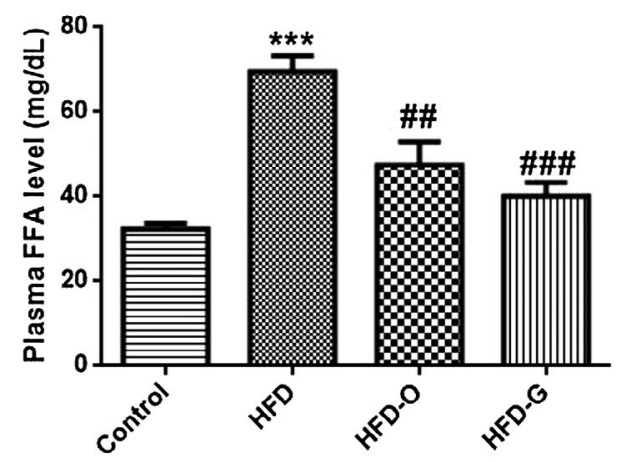

(f)

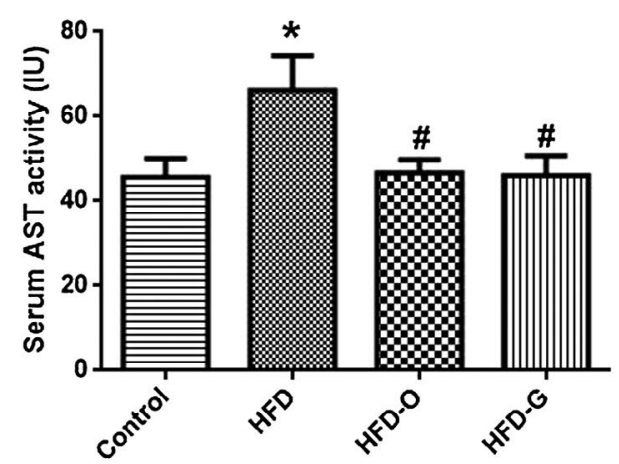

FIG URE 5 Plasma biochemical data. (a) Plasma glucose, (b) plasma total cholesterol, (c) plasma triglycerides, (d) plasma free fatty acids, (e) plasma ALT, (f) plasma AST. Data are represented as means \pm SE. HFD means are statistically significant at $\left({ }^{*}\right) p \leq .1,\left({ }^{* *}\right) p \leq .05,\left(\left(^{* * *}\right) p \leq .001\right.$ compared to control group. HFD-O and HFD-G means are statistically significant at (\#) $p \leq .1$, (\#\#) $p \leq .05$, (\#\#\#) $p \leq .001$ compared to HFD group. HFD, high-fat diet group; HFD-O, high-fat diet group treated with omega-3 fatty acids; HFD-G, high-fat diet group treated with Zingiber officinale extract

hepatic enzymes. The increase in hepatic enzymes AST and ALT were significant compared to normal healthy rats which are a key marker for NAFLD pathogenesis (Lai et al., 2016). The HFD rat's liver appeared pale in color with an accompanying abnormal histological accumulation of fat in hepatocytes. The effect shown in our biochemical and histological data is coherent with other studied NAFLD models (Rodriguez-Ramiro et al., 2016) indicating that the diet used was successful to produce a classical NAFLD model for performing the current study. The treatment of rats with Z. officinale or omega-3 reversed all biochemical and genetic dysregulation seen in HFD-fed rats. There was a decrease in blood glucose, TG, and TC indicating that there was less fat load from blood on the liver. This correction of plasma parameters is consistent with the treated rat's resolved liver morphology and liver H\&E-stained sections. It was clear that the cells closely surrounding the central vein and more nourished with $Z$. officinale or omega-3 were almost free from abnormal fat accumulation compared to cells in the section periphery, supporting the beneficial effect of both therapies. The therapeutic effect demonstrated 
(a)

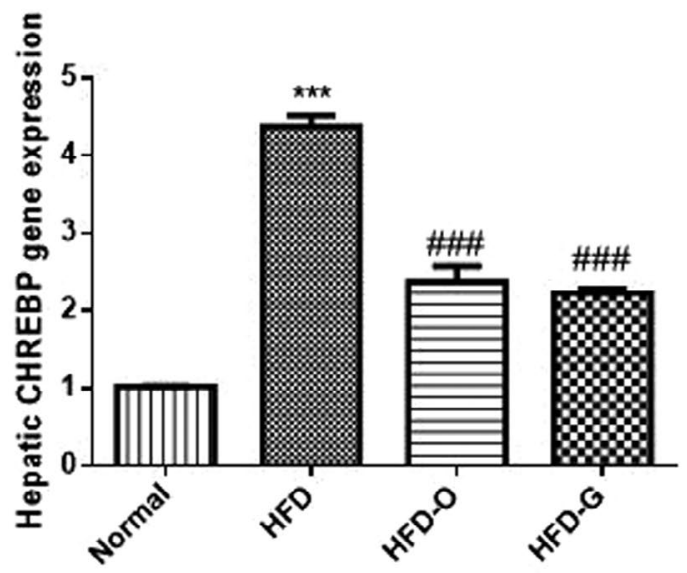

(c)

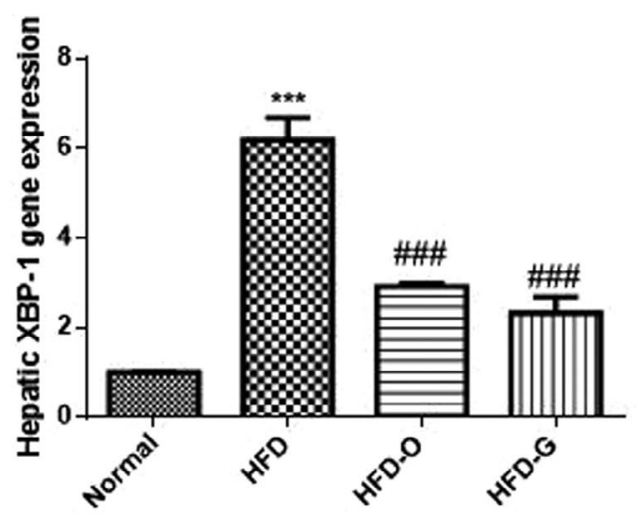

(b)

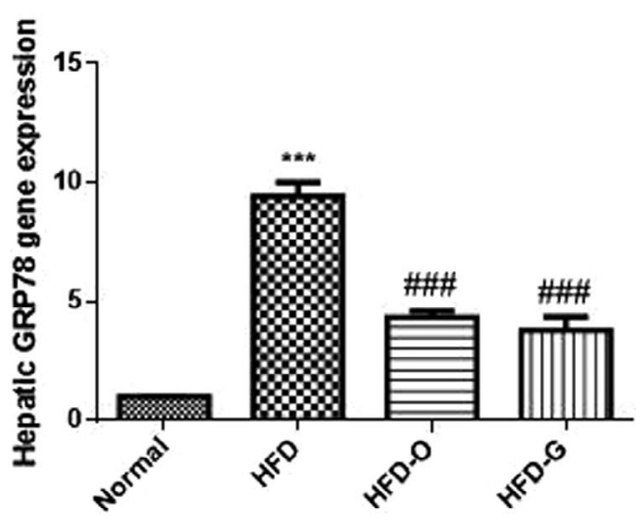

(d)

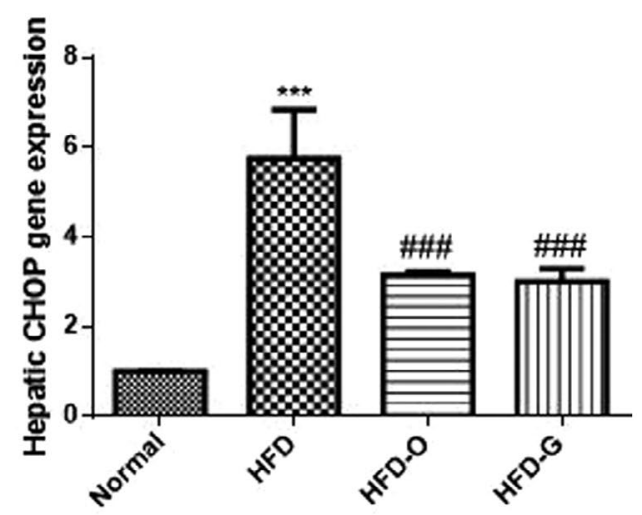

FIGURE 6 Relative hepatic gene expression. Data are represented as means \pm SE. HFD means are statistically significant at $\left({ }^{*}\right) p \leq .1$, $\left.{ }^{(* *}\right) p \leq .05,\left({ }^{* * *}\right) p \leq .001$ compared to control group. HFD-O and HFD-G means are statistically significant at (\#) $p \leq .1,(\# \#) p \leq .05$, (\#\#\#) $p \leq .001$ compared to HFD group. HFD, high-fat diet group; HFD-O, high-fat diet group treated with omega-3 fatty acids; HFD-G, high-fat diet group treated with Zingiber officinale extract

by Z. officinale or omega-3 is coherent with other studies that evaluated both therapies or their individual components against metabolic syndrome disorders such as type 2 diabetes, obesity, IR, and NAFLD. These effects have been studied to be through regulation of lipogenesis, $\beta$-oxidation besides their antioxidant and anti-inflammatory activity (de Castro, Cardoso, Calder, Jordão, \& Vannucchi, 2015; Huang, Deng, Meng, \& Ma, 2019; Lai et al., 2016; Li et al., 2014; Molinar-Toribio et al., 2015; Singh, Akanksha, Singh, Maurya, Srivastava, 2009). Here, we demonstrated a novel pathway by which the therapeutic approaches treat NAFLD by modulation of ER stress proposed to be responsible for NAFLD progression through different mechanisms (Ferré \& Foufelle, 2010; Jo et al., 2013; Lee et al., 2012).

The hyperglycemia and hyperlipidemic state induced by HFD feeding contributed to the induction of hepatic glucose-sensitive ChREBP. Increase in ChREBP induce hepatic lipogenic genes and promote hepatic steatosis (S. Bin Lee et al., 2016). Moreover, in our experimental model, HFD-fed rats showed increased ER stress markers CHOP, XBP1, and GRP78 which is coherent with previously reported findings linking ER stress with different metabolic disorders (Jo et al., 2013; Lee et al., 2012). ER stress is known to activate hepatic de novo lipogenesis, inhibit VLDL secretion, promote IR and inflammatory process, and promote cell apoptosis through pathways including CHOP/Caspase. ER stress increases the level of fat accumulation and worsens the NAFLD to a more serious hepatic state (Ferré \& Foufelle, 2010; Jang et al., 2016). Studies showed that CHOP deletion protected from diet-induced steatohepatitis (Tamaki et al., 2008). Thereby, drug-dependent relieving of ER stress and decreasing ER stress-associated markers represent an eligible therapeutic goal for the treatment of NAFLD (Kammoun, Hainault, Ferré, \& Foufelle, 2009). A previous study showed that ameliorating ER stress using Schisandra Chinensis herbal medicine has resolved hepatic steatosis in mice and in HepG2 cells (Jang et al., 2016) similarly to our proposed NAFLD rat model. This indicates that reducing ER stress through $Z$. officinale and omega- 3 fatty acids could be the way to treat fatty liver.

Both therapeutic approaches reduced hepatic expression for ER stress markers CHOP, XBP1, and GRP78 with non-significant more efficacy for the Z. officinale extract over omega-3 fatty acids. Z. officinale and omega- 3 fatty acids also prevented ER stress through 

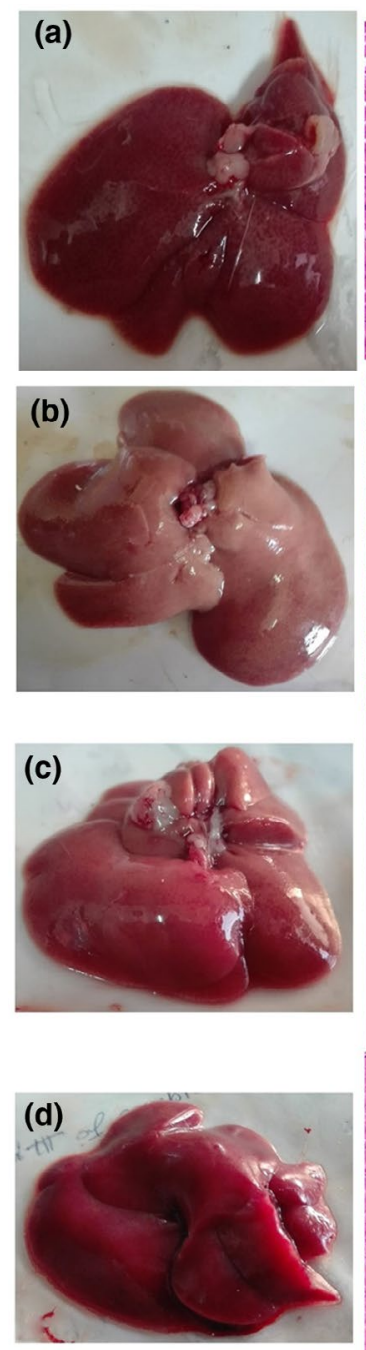
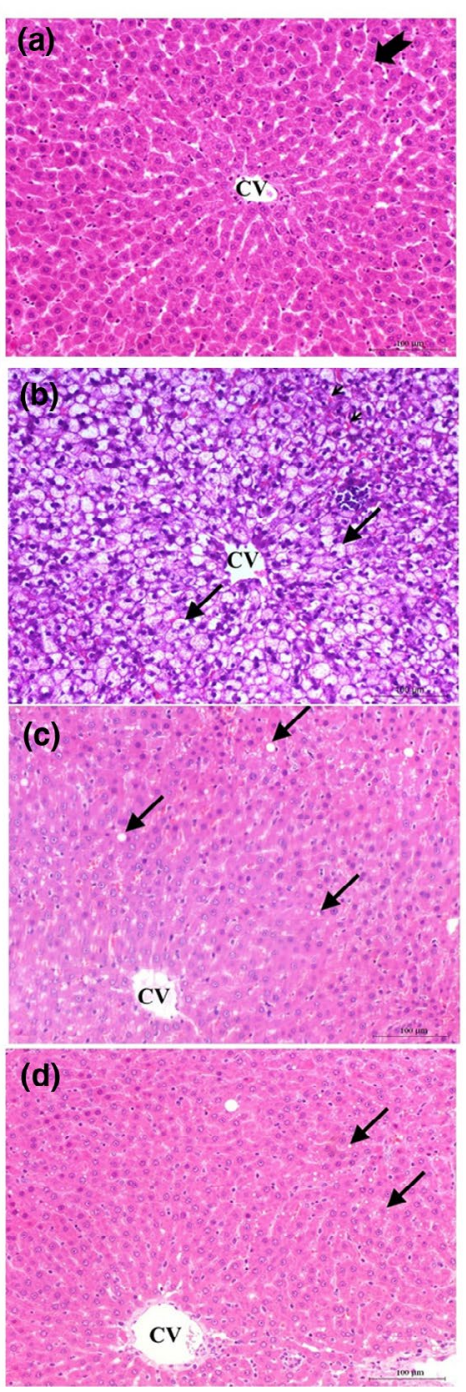

FIGURE 7 Liver morphology besides H\&E for (a) control group, (b) high-fat diet group, (c) high-fat diet group treated with omega-3 fatty acids, (d) high-fat diet group treated with Zingiber officinale extract. Central vein (CV), normal hepatocytes (notched arrow), congested sinusoids (arrows heads), minute micro-vesicular steatosis (line arrows)

their inhibition of ChREBP lipogenic gene either directly or through reducing blood glucose level.

The combined results represented in this study proves that our therapeutic strategy not only decreased ER stress and the resultant intrahepatic fat accumulation preventing NAFLD progression to a more serious case, but also it reverted liver into a normal healthy state with non-significant parameters compared to normal control rats (Adams \& Angulo, 2006).

\section{5 | CONCLUSION}

From this study, we demonstrated that $Z$. officinale extract at a slightly more efficacy than omega-3 fatty acids attenuate ER stress associated with NAFLD. This effect and the direct inhibition

of hepatic lipogenesis prevented the pathogenesis of hepatic steatosis and reverted liver to normal hemostasis. Z. officinale extract is a potential therapeutic agent for the treatment of NAFLD besides targeting other ER stress-associated diseases in the liver and other organs.

\section{ACKNOWLEDGMENTS}

We acknowledge Professor Dr. Laila A. Rashed, Department of Biochemistry and Molecular Biology, Faculty of Medicine, Cairo University for performing $\mathrm{qPCR}$ and western blotting analysis included in this study. We also acknowledge Professor Dr. Khalid M. Mazhar, Department of Pathology, Faculty of Veterinary Medicine, Beni-Suef University for his valuable help in the performance of the histopathological tests. We acknowledge Scientific illustration for the research scientist website for providing free sketch material for designing Figure 2 (graphical abstract).

\section{CONFLICT OF INTEREST}

Authors declare that they have no conflicts of interest.

\section{ORCID}

Mohamed A. Tohamy iD https://orcid.org/0000-0002-5232-4766

\section{REFERENCES}

Adams, L. A., \& Angulo, P. (2006). Treatment of non-alcoholic fatty liver disease. Postgraduate Medical Journal, 82(967), 315-322. https://doi. org/10.1136/pgmj.2005.042200

Bhandari, U., Kanojia, R., \& Pillai, K. K. (2005). Effect of ethanolic extract of Zingiber officinale on dyslipidaemia in diabetic rats. Journal of Ethnopharmacology, 97(2), 227-230. https://doi.org/10.1016/j. jep.2004.11.011

Bhargava, S., Dhabhai, K., Batra, A., Sharma, A., \& Malhotra, B. (2012). Zingiber Officinale: Chemical and phytochemical screening and evaluation of its antimicrobial activities. Journal of Chemical and Pharmaceutical Research, 4(1), 360-364.

Browning, J. D., \& Horton, J. D. (2004). Molecular mediators of hepatic steatosis and liver injury. The Journal of Clinical Investigation, 114(2), 147-152. https://doi.org/10.1172/JCl200422422.

Buzzetti, E., Pinzani, M., \& Tsochatzis, E. A. (2016). The multiplehit pathogenesis of non-alcoholic fatty liver disease (NAFLD). Metabolism: Clinical and Experimental, 65(8), 1038-1048. https://doi. org/10.1016/j.metabol.2015.12.012

de Castro, G. S., Cardoso, J. F. R., Calder, P. C., Jordão, A. A., \& Vannucchi, H. (2015). Fish oil decreases hepatic lipogenic genes in rats fasted and refed on a high fructose diet. Nutrients, 7(3), 1644-1656. https:// doi.org/10.3390/nu7031644

Denny Joseph, K. M., \& Muralidhara, M. (2012). Fish oil prophylaxis attenuates rotenone-induced oxidative impairments and mitochondrial dysfunctions in rat brain. Food and Chemical Toxicology, 50(5), 15291537. https://doi.org/10.1016/j.fct.2012.01.020

Dongiovanni, P., Lanti, C., Riso, P., \& Valenti, L. (2016). Nutritional therapy for nonalcoholic fatty liver disease. The Journal of Nutritional Biochemistry, 29, 1-11. https://doi.org/10.1016/J.JNUTB IO.2015.08.024 
Ferré, P., \& Foufelle, F. (2010). Hepatic steatosis: A role for de novo lipogenesis and the transcription factor SREBP-1c. Diabetes, Obesity and Metabolism, 12(Suppl 2), 83-92. https://doi. org/10.1111/j.1463-1326.2010.01275.x

Huang, F., Deng, T., Meng, L., \& Ma, X. (2019). Dietary ginger as a traditional therapy for blood sugar control in patients with type 2 diabetes mellitus. Medicine, 98(13), e15054. https://doi.org/10.1097/ MD.0000000000015054

Itaya, K. (1977). A more sensitive and stable colorimetric determination of free fatty acids in blood. Journal of Lipid Research, 18(7), 663-665.

Jang, M.-K., Nam, J. S., Kim, J. H., Yun, Y.-R., Han, C. W., Kim, B. J., ... Jung, M. H. (2016). Schisandra chinensis extract ameliorates nonalcoholic fatty liver via inhibition of endoplasmic reticulum stress. Journal of Ethnopharmacology, 185, 96-104. https://doi.org/10.1016/j. jep.2016.03.021

Jo, H., Choe, S. S., Shin, K. C., Jang, H., Lee, J. H., Seong, J. K., ... Kim, J. B. (2013). Endoplasmic reticulum stress induces hepatic steatosis via increased expression of the hepatic very low-density lipoprotein receptor. Hepatology, 57(4), 1366-1377. https://doi.org/10.1002/ hep. 26126

Kammoun, H. L., Hainault, I., Ferré, P., \& Foufelle, F. (2009). Nutritiona related liver disease: Targeting the endoplasmic reticulum stress. Current Opinion in Clinical Nutrition and Metabolic Care, 12(6), 575582. https://doi.org/10.1097/MCO.0b013e32833189db

Lai, Y.-S., Lee, W.-C., Lin, Y.-E., Ho, C.-T., Lu, K.-H., Lin, S.-H., ... Sheen, L.-Y. (2016). Ginger essential oil ameliorates hepatic injury and lipid accumulation in high fat diet-induced nonalcoholic fatty liver disease. Journal of Agricultural and Food Chemistry, 64(10), 2062-2071. https://doi.org/10.1021/acs.jafc.5b06159

Lee, J.-S., Zheng, Z., Mendez, R., Ha, S.-W., Xie, Y., \& Zhang, K. (2012). Pharmacologic ER stress induces non-alcoholic steatohepatitis in an animal model. Toxicology Letters, 211(1), 29-38. https://doi. org/10.1016/J.TOXLET.2012.02.017

Lee, S. B., Cho, H. I., Jin, Y. W., Lee, E. K., Ahn, J. Y., \& Lee, S. M. (2016). Wild ginseng cambial meristematic cells ameliorate hepatic steatosis and mitochondrial dysfunction in high-fat diet-fed mice. Journal of Pharmacy and Pharmacology, 68(1), 119-127. https://doi. org/10.1111/jphp.12487

Li, Y., Tran, V. H., Kota, B. P., Nammi, S., Duke, C. C., \& Roufogalis, B. D. (2014). Preventative effect of Zingiber officinale on insulin resistance in a high-fat high-carbohydrate diet-fed rat model and its mechanism of action. Basic and Clinical Pharmacology and Toxicology, 115(2), 209215. https://doi.org/10.1111/bcpt.12196

Marsman, H. A., Heger, M., Kloek, J. J., Nienhuis, S. L., van Werven, J. R., Nederveen, A. J., ... van Gulik, T. M. (2011). Reversal of hepatic steatosis by omega-3 fatty acids measured non-invasively by $1 \mathrm{H}$-magnetic resonance spectroscopy in a rat model. Journal of Gastroenterology and Hepatology (Australia), 26(2), 356-363. https:// doi.org/10.1111/j.1440-1746.2010.06326.x

Mazidi, M., Katsiki, N., \& Banach, M. (2019). A higher flavonoid intake is associated with less likelihood of nonalcoholic fatty liver disease: Results from a multiethnic study. Journal of Nutritional Biochemistry, 65, 66-71. https://doi.org/10.1016/j.jnutbio.2018.10.001

Molinar-Toribio, E., Pérez-Jiménez, J., Ramos-Romero, S., Romeu, M. Giralt, M., Taltavull, N., ... Torres, J. L. (2015). Effect of n-3 PUFA supplementation at different EPA:DHA ratios on the spontaneously hypertensive obese rat model of the metabolic syndrome. British Journal of Nutrition, 113(6), 878-887. https://doi.org/10.1017/S0007 114514004437

Nammi, S., Sreemantula, S., \& Roufogalis, B. D. (2009). Protective effects of ethanolic extract of Zingiber Officinale rhizome on the development of metabolic syndrome in high-fat diet-fed rats. Basic and Clinical Pharmacology and Toxicology, 104(5), 366-373. https://doi. org/10.1111/j.1742-7843.2008.00362.x

National Research Council. (2011). Guide for the care and use of laboratory animals (8th ed.). Washington, DC: The National Academies Press. https://doi.org/10.17226/12910
Pan, F., Su, T. J., Cai, S. M., \& Wu, W. (2017). Fungal endophyte-derived Fritillaria unibracteata var. wabuensis: Diversity, antioxidant capacities in vitro and relations to phenolic, flavonoid or saponin compounds. Scientific Reports, 7(211), 1-14. https://doi.org/10.1038/ srep42008

Pisonero-Vaquero, S., Martínez-Ferreras, Á., García-Mediavilla, M. V., Martínez-Flórez, S., Fernández, A., Benet, M., ... Sánchez-Campos, S. (2015). Quercetin ameliorates dysregulation of lipid metabolism genes via the PI3K/AKT pathway in a diet-induced mouse model of nonalcoholic fatty liver disease. Molecular Nutrition and Food Research, 59(5), 879-893. https://doi.org/10.1002/mnfr.20140 0913

Rahmani, A. H., Al Shabrmi, F. M., \& Aly, S. M. (2014). Active ingredients of ginger as potential candidates in the prevention and treatment of diseases via modulation of biological activities. International Journal of Physiology, Pathophysiology \& Pharmacology, 6(2), 125-136. https:// doi.org/10.1016/0029-7844(94)00457-O

Rodriguez-Ramiro, I., Vauzour, D., \& Minihane, A. M. (2016). Polyphenols and non-alcoholic fatty liver disease: Impact and mechanisms. Proceedings of the Nutrition Society, 75(1), 47-60. https://doi. org/10.1017/S0029665115004218

Saponaro, C., Gaggini, M., \& Gastaldelli, A. (2015). Nonalcoholic fatty liver disease and type 2 diabetes: Common pathophysiologic mechanisms. Current Diabetes Reports, 15(6), 1-13. https://doi.org/10.1007/ s11892-015-0607-4

Singh, A. B., Akanksha, J., Singh, N., Maurya, R., \& Srivastava, A. K. (2009). Anti-hyperglycaemic, lipid lowering and anti-oxidant properties of [6]-gingerol in $\mathrm{db} / \mathrm{db}$ mice. International Journal of Medicine and Medical Sciences, 1(12), 536-544.

Stanković, M. S. (2011). Total phenolic content, flavonoid concentration and antioxidant activity of Marrubium peregrinum L. extracts. Kragujevac Journal of Science, 33, 63-72. http://doi. $\operatorname{org} / 581.19: 582.929 .4: 577.164 .3$

Tamaki, N., Hatano, E., Taura, K., Tada, M., Kodama, Y., Nitta, T., ... Uemoto, S. (2008). CHOP deficiency attenuates cholestasis-induced liver fibrosis by reduction of hepatocyte injury. American Journal of Physiology-Gastrointestinal and Liver Physiology, 294(2), G498-G505. https://doi.org/10.1152/ajpgi.00482.2007

Tirosh, O. (2014). Liver metabolism and fatty liver disease. Retrieved from https://books.google.com/books?hl=en\&lr=\&xml:id=GxObBAAAQB AJ\&pgis=1

Venkat Kullai Setty, N., Santhosh, D., Narasimha Rao, D., Sanjeeva Kumar, A., Charles Martin, A., \& Shri Lakshmi Narasimha College. (2011). Preliminary phytochemical screening and antidiabetic activity of Zingiber officinale rhizomes. International Journal of Pharmacy and Life Sciences, 2(12), 1287-1292.

Wang, J., Ke, W., Bao, R., Hu, X., \& Chen, F. (2017). Beneficial effects of ginger Zingiber officinale Roscoe on obesity and metabolic syndrome: A review. Annals of the New York Academy of Sciences, 1398(1), 83-98. https://doi.org/10.1111/nyas.13375

Younossi, Z. M., Koenig, A. B., Abdelatif, D., Fazel, Y., Henry, L., \& Wymer, M. (2016). Global epidemiology of nonalcoholic fatty liver diseasemeta-analytic assessment of prevalence, incidence, and outcomes. Hepatology, 64(1), 73-84. https://doi.org/10.1002/hep.28431

Zhang, Y., Xue, R., Zhang, Z., Yang, X., \& Shi, H. (2012). Palmitic and linoleic acids induce ER stress and apoptosis in hepatoma cells. Lipids in Health and Disease, 11, 1. https://doi.org/10.1186/1476-511X-11-1

How to cite this article: Kandeil MA, Hashem RM, Mahmoud $\mathrm{MO}$, Hetta MH, Tohamy MA. Zingiber officinale extract and omega-3 fatty acids ameliorate endoplasmic reticulum stress in a nonalcoholic fatty liver rat model. J Food Biochem. 2019;43:e13076. https://doi.org/10.1111/jfbc.13076 